\title{
Spherical refractive correction with an electro-optical liquid lens in a double-pass system
}

\section{F. Sanàbria \\ ferran.sanabria@upc.edu}

\section{F. Díaz-Doutón}

\section{Aldaba}

J. Pujol

\begin{abstract}
Centre for Sensors, Instruments and Systems Development, Universitat Politècnica de Catalunya, Rambla Sant Nebridi 10, 08222 Terrassa, Spain

Centre for Sensors, Instruments and Systems Development, Universitat Politècnica de Catalunya, Rambla Sant Nebridi 10, 08222 Terrassa, Spain
\end{abstract}

Centre for Sensors, Instruments and Systems Development, Universitat Politècnica de Catalunya, Rambla Sant Nebridi 10, 08222 Terrassa, Spain

Centre for Sensors, Instruments and Systems Development, Universitat Politècnica de Catalunya, Rambla Sant Nebridi 10, 08222 Terrassa, Spain

In this study we show a novel double-pass configuration to correct the spherical refractive error by means of an electro-optical liquid lens. The proposed method enables spherical correction in the $-12 D$ to $+7 \mathrm{D}$ range without movable parts using an electro-optical liquid lens. We have measured the optical performance of the spherical corrector in terms of power, pupil size and optical quality verifying that it fits the requirements to be applied to a double-pass system. We have also evaluated the performance of the proposed method in patients by comparison with a conventional double-pass system using a Badal optometer.

[DOI: http://dx.doi.org/10.2971/jeos.2013.13062]

Keywords: Electro-optical liquid lens, tuneable lens, optical quality, eye, refraction

\section{INTRODUCTION}

The double-pass technique, based on recording images of a point-source object after reflection on the retina and a double pass through the ocular media [1] is a powerful tool to evaluate the optical quality of the eye. It has been widely used in studies assessing the performance of the eye in different conditions, such as age-related changes in optical quality [2] and in clinical studies comparing the performance of phakic IOLs with LASIK surgery [3].

The main aberration that degrades the quality of the retinal image is defocus, followed by astigmatism [4]. When measuring the optical quality of the eye with a double-pass technique, these low order aberrations are usually corrected. In this way, their contribution does not mask the effect of higher order aberrations. The most common method to correct astigmatism is through trial lenses, whereas Badal optometers are used for defocus correction [2].

The Badal optometer is based on the change in optical path between two lenses in order to achieve the desired vergence at the exit of the optometer. It usually consists of two movable mirrors located between both lenses. The movement of the mirrors changes the length of the optical path between the lenses, resulting in different defocus corrections at the exit of the system. The Badal optometer is affordable and simple, and permits a wide range of spherical correction just by manipulating a couple of mirrors and maintaining the rest of the setup fixed. Despite these advantages, the weak point of the Badal system are its movable components, which limit the speed of the response and increase the setup dimensions.

In the past few years, focusable electro-optical liquid lenses have been developed. These lenses consist of a liquid element covered by an elastic membrane. When voltage is applied to the lens, the curvature of the surface changes, thus modifying the spherical power of the lens. These lenses have been applied to different uses: to change the focal of the microlens array in a Hartmann-Shack system [5] and as an adaptive optics actuator in different applications such as optical coherence microscopy [6].

In this study we present a method for spherical correction based on the use of an electro-optical liquid lens. We consider that in comparison with the Badal optometer, the proposed method offers the following advantages: the electro-optical liquid lens based spherical corrector is faster than a Badal optometer and it does not have any movable parts; it is also more compact and much easier to align. Consequently, this is a workable method for correcting spherical error in doublepass or similar systems.

\section{MATERIAL AND METHODS}

\subsection{Setup}

The method proposed is shown in Figure 1. The first pass is shown in blue, whereas the second pass is shown in orange. 


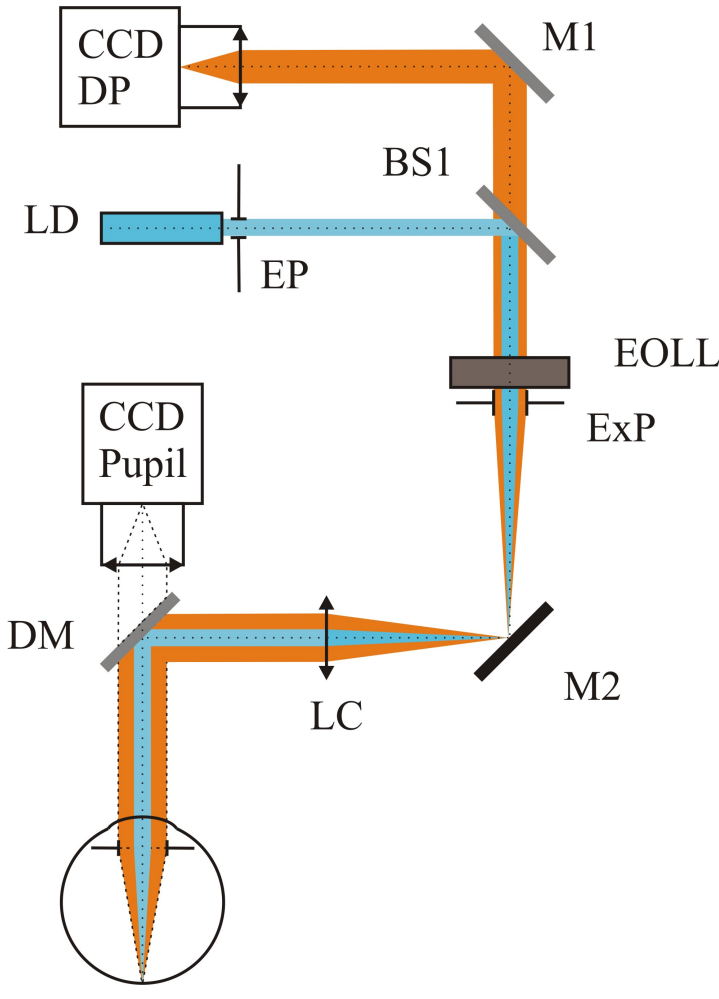

FIG. 1 Scheme of the double-pass setup: CCD cameras (CCD-DP a CCD-Pupil), mirrors (M1 \& M2), beam splitter (BS), laser diode (LD), entrance and exit pupils (EP \& ExP), electro-optical liquid lens (EOLL), compensating lens (LC) and dichroic mirror (DM).

Like in a conventional double-pass configuration, a collimated laser diode (LD) is the light source; this light passes through a diaphragm acting as entrance pupil (EP) of the system. After reflection in a beam splitter (BS1), the light reaches the electro-optical liquid lens (EOLL) and then it passes through a compensating lens (LC). This compensating lens (LC) is introduced into the system in order to shift the focal range of the electro-optical liquid lens (EOLL) to our working focal range. Moreover, this lens (LC) is used to ensure that the diaphragm acting as exit pupil (ExP) is conjugated with the pupil of the patient. Next, the light is reflected in a dichroic mirror (DM) and reaches the eye of the patient. After retinal reflection, the second pass begins, with an optical path identical to the first pass until BS1. Afterwards, the second pass differs from the first and the image is recorded in a CCD camera after reflection in a mirror (M1). The entrance and exit pupils used in this setup were of 2 and $4 \mathrm{~mm}$ respectively as this is a extensively applied in basic research and clinical studies of the optics of the eye $[10,11]$. This asymmetric configuration enables obtaining the information of all the aberrations in the eye [8] and the results obtained with the $4 \mathrm{~mm}$ exit pupil are a good descriptor of the average optical performance of the eye.

The spherical corrector consists in the electro-optical liquid lens and the compensating lens. We have used the commercial Fast Electrically Tunable Lens EL-10-30 (Optotune) with a focal range from +36 to $+132 \mathrm{~mm}$. The focal of the LC compensating lens has been set at $+50 \mathrm{~mm}$. The distance between both lenses is twice the focal of the LC lens, as well as the distance between the LC and the eye's pupil plane.

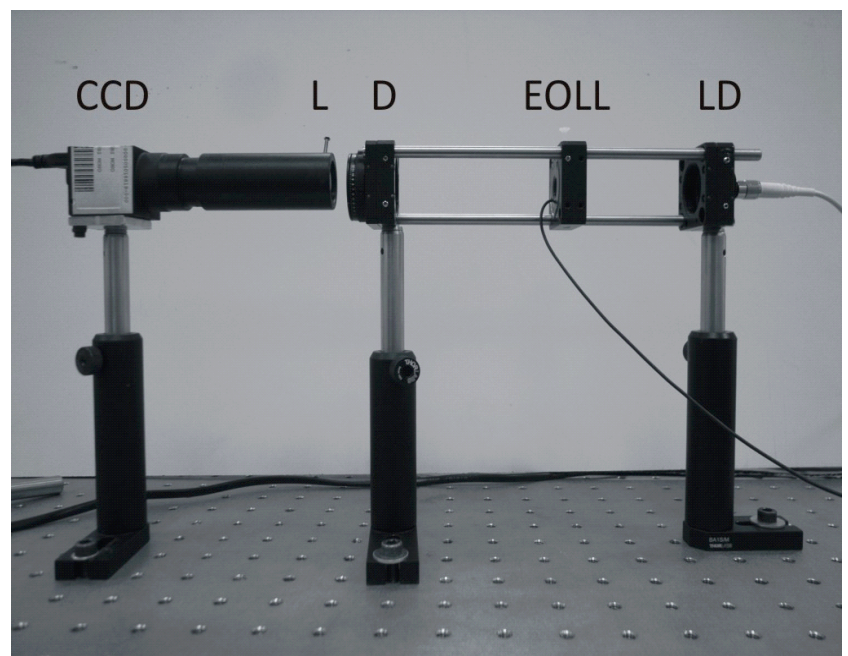

FIC. 2 Single-pass setup for the optical quality measurement of the electro-optical liquid lens. CCD camera, objective lens (L), diaphragm (D), electro-optical liquid lens (EOLL) and laser diode (LD).

\subsection{Optical quality of the electro-optical liquid lens}

We measured the optical quality of the electro-optical liquid lens so as to prove its usefulness in the measurement of the eyes optical quality. For this purpose, we built a simple singlepass setup, shown in Figure 2. A laser diode (LD) is collimated by the electro-optical liquid lens (EOLL) and passes through a diaphragm with a diameter of $4 \mathrm{~mm}$, as is the pupil used in our system. The image is recorded by a CCD by means of a $100 \mathrm{~mm}$ lens that behaves as an objective (L). We used the Strehl ratio as the parameter to evaluate the optical quality. The measurements were performed for the focal of the electrooptical liquid lens from 40 to $100 \mathrm{~mm}$ in $10 \mathrm{~mm}$ steps, with 3 repetitions for each of them.

\subsection{Measurements in patients}

Finally, we measured the performance of the proposed setup in real patients. 9 patients with a mean age of $36.22 \pm 8.71$ years (range: 24 to 48 ) and a mean spherical refraction of $-1.33 \pm 2.45 \mathrm{D}$ (range: 1.25 to -4.5 ) were enrolled in the study. The optical quality measured with our setup including an electro-optical liquid lens for spherical refractive correcction, was compared with the results obtained with a commercial double-pass system (OQAS, Visiometrics S.L. [7]) using a Badal optometer for spherical correction.

\section{RESULTS AND DISCUSSION}

\subsection{Setup}

With the configuration shown in Figure 1 the corrector power ranges from +7.77 to $-12.42 \mathrm{D}$ and results in a $1: 1$ relation between the change in the electro-optical liquid lens and the spherical corrector power. Figure 3 shows the power of the electro-optical liquid lens alone (red) and the power of the spherical corrector including the electro-optical and compensating lens (blue) against the applied electrical intensity. Using 


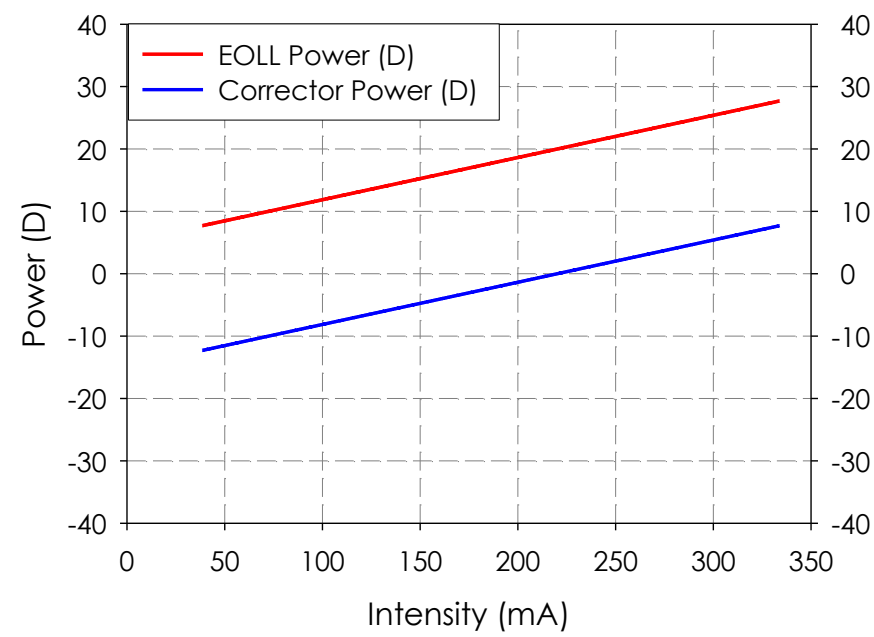

FIG. 3 Power in diopters (D) of the electro-optical liquid lens alone (red) and the power of the spherical corrector including the electro-optical and compensating lens (blue), against the applied electrical intensity in milliamperes $(\mathrm{mA})$.

a different focal for the compensating lens shifts the curve of the spherical corrector.

If the distance between the electro-optical liquid lens and the compensating lens is changed from twice the LC focal, the relation between the power of the electro-optical liquid lens and the spherical corrector power changes. The range of the spherical corrector can be extended using longer distances resulting in a loss of resolution, whereas a corrector with higher resolution is obtained for shorter distances, resulting in a range loss. Due to curvature and consequent thickness changes in the electro-optical liquid lens when varying the focal, the distance between the principal planes of the electro-optical liquid lens and the compensating lens is not constant for all cases, which causes a small error in the relation between the electrooptical liquid lens and the spherical compensator power. With the specifications given by the manufacturer of the electrooptical liquid lens used, we estimated a maximum movement of the principal planes of $3 \mathrm{~mm}$. With this data the error concerning the ideal calculation for the extreme cases $(-12 \mathrm{D}$ and $+7 \mathrm{D})$ was $-0.009 \mathrm{D}$ and $+0.047 \mathrm{D}$, respectively. This error is easily solvable by considering the exact spherical correction of the system in function of the intensity applied to the electrooptical liquid lens.

Moreover, the distance between the electro-optical liquid lens and the compensating lens is important for the correct size and position of the systems pupils. In the double-pass technique, entrance and exit pupils are important elements that affect the final image quality [8,9]. It is therefore crucial to control these variables. The proposed configuration, with the distance between those lenses being twice the focal of LC, and equal to the distance between LC and the eyes pupil plane, ensures a constant magnification of 1 for all the power range. On the other hand, when using different distances between lenses, the magnification changes in relation to the different powers of the range. Similarly to spherical power, curvature and thickness changes in the electro-optical liquid lens to achieve the different focal lengths do not allow to keep the correct distance between the principal planes of the electro-optical liquid and the compensating lens. Conse-

\begin{tabular}{|c|c|c|}
\hline $\begin{array}{c}\text { Focal length } \\
(\mathbf{m m})\end{array}$ & Mean Strehl & SD \\
\hline 40 & 0.790 & 0.026 \\
50 & 0.833 & 0.026 \\
60 & 0.814 & 0.011 \\
70 & 0.833 & 0.022 \\
80 & 0.832 & 0.009 \\
90 & 0.836 & 0.003 \\
100 & 0.820 & 0.007 \\
\hline
\end{tabular}

TABLE 1 Strehl ratio of the electro-optical liquid lens for the different focal lengths measured with $780 \mathrm{~nm}$ laser diode and aperture diameter of $4 \mathrm{~mm}$.

quently, there is a magnification change for the different powers of the spherical corrector. Considering the specifications given by the manufacturer, we found a small error in the size of the entrance and exit pupils of the system. The most commonly used double-pass systems have a small entrance pupil of $2 \mathrm{~mm}$. In this case, the error in diameter for $+7 \mathrm{D}$ and -12 D corrections were $0.01 \mathrm{~mm}$ and $0.02 \mathrm{~mm}$, respectively. For an exit pupil of $4 \mathrm{~mm}$, the error for $+7 \mathrm{D}$ and $-12 \mathrm{D}$ was $0.07 \mathrm{~mm}$ and $0.08 \mathrm{~mm}$, respectively. In our view, these error values in the entrance and exit pupils are irrelevant when measuring the optical quality of the eye.

\section{2 optical quality of the electro-optical liquid lens}

In Table 1, the resulting Strehl ratios and their standard deviations measured with the single-pass setup shown in Figure 2 are shown for the different measured focal lengths. The Strehl ratio of the electro-optical liquid lens (around 0.8 for all focal lengths) was much higher than the mean Strehl ratio of the eye, with a value around $0.25[2,10]$. Thus, despite the slight optical degradation induced by the electro-optical liquid lens, it is negligible compared with the quality of the eye. However it has to be taken into account that according to the manufacturer specifications, the performance of the EOLL for large pupils $(8 \mathrm{~mm})$ decreases due to an effect of the gravity in the membrane deformation and could induce a significant error when measuring the eye's optical quality for large pupils. As the manufacturer suggest, this can be minimized by placing the lens horizontally.

\subsection{Measurements in patients}

Finally, we compared the optical quality measured in patients with a conventional double-pass system where the refraction is corrected by means of a Badal system and our experimental setup using an electro-optical liquid lens. We used the cutoff frequency of the MTF (MTFcutoff), commonly used in clinical practice, as the optical quality parameter. The mean MTFcutoff value for all patients measured with the OQAS system and the setup was of $34.56 \pm 9.39$ cycles per degress (c/deg) and $35.24 \pm 9.86 \mathrm{c} / \mathrm{deg}$ respectively, with a mean difference of $0.68 \pm 2.18 \mathrm{c} / \mathrm{deg}$. In Figure 4 the correlation between the MTFcutoff obtained with the OQAS system and the experimental setup is shown, with a Pearson correlation coefficient of 0.975 and statistically significant $(\mathrm{p}<0.01)$. Finally, after confirming the normal distribution of the measurements 


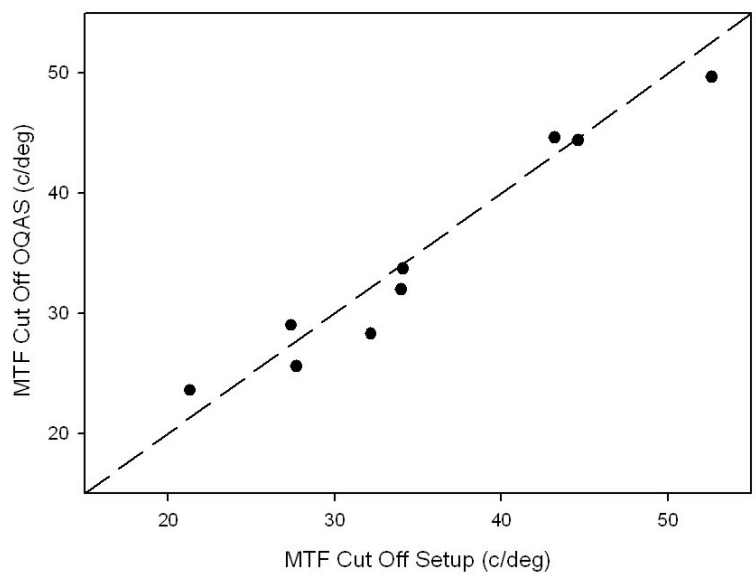

FIC. 4 Correlation of the MTFcutoff obtained with the OQAS system and the experimental setup.

the $\mathrm{t}$-Test showed no statisitcally significant differences ( $p=0.375)$ between both methods. Thus, from all this data we can conclude that the performance of the double-pass system based on the electro-optical liquid lens is similar to a double-pass system based on a Badal optometer, when testing it in real patients with a wide range of spherical refraction.

In conclusion, we have shown the suitability of an electrooptical liquid lens based spherical correction to be used in a double-pass system. We consider the limitations of the electrooptical liquid lens regarding pupil size magnification for different focal lengths and optical quality degradation as minor problems of this system. However, the use of an electro-optical liquid lens offers the advantage of a faster response, lack of movable parts and a smaller size. Consequently, it is a valid solution for the spherical correction in double-pass or similar systems such as Hartmann-Shack sensors.

\section{ACKNOWLEDGEMENTS}

This study was funded by the Spanish Ministry of Science and Innovation (project grant DPI2011-30090-C02-01) and the European Union.

\section{References}

[1] J. Santamaría, P. Artal, and J. Bescós, "Determination of the pointspread function of human eyes using a hybrid optical-digital method," J. Opt. Soc. Am. A 4, 1109-1114 (1987).

[2] A. Guirao, C. González, M. Redondo, E. Geraghty, S. Norrby, and P. Artal, "Average optical performance of the human eye as a function of age in a normal population," Invest. Ophthalmol. Vis. Sci. 40, 203-213 (1999).

[3] M. Vilaseca, A. Padilla, J. Pujol, J. C. Ondategui, P. Artal and J. L. Cüell, "Optical quality one month after verisyse and Veriflex phakic IOL implantation and Zeiss MEL 80 LASIK for myopia from 5.00 to 16.50 diopters," J. Refract. Surg. 25, 689-698 (2009).

[4] J. F. Castejón-Mochón, N. López-Gil, A. Benito, and P. Artal, “Ocular wave-front aberration statistics in a normal young population," Vision Res. 42, 1611-1617 (2002).

[5] R. Martínez-Cuenca, V. Durán, V. Climent, E. Tajahuerce, S. Bará , J. Ares, J. Arines, M. Martínez-Corral, and J. Lancis, "Reconfigurable Shack-Hartmann sensor without moving elements," opt. Lett. 35, 1338-1340 (2010).

[6] S. Murali , P. Meemon, K. S. Lee, W. P. Kuhn, K. P. Thompson, and J. P. Rolland, "Assessment of a liquid lens enabled in vivo optical coherence microscope," Appl. Optics 49, 145-156 (2010).

[7] J. L. Güell, J. Pujol, M. Arjona, F. Diaz Douton, and P. Artal, “Optical Quality Analysis System: Instrument for objective clinical evaluation of ocular optical quality," J. Cataract Refract. Surg. 30, 15981599 (2004).

[8] P. Artal, I. Iglesias, N. López-Gil, and D. G. Green, “Double-pass measurements of the retinal-image quality with unequal entrance and exit pupil sizes and the reversibility of the eye's optical system," J. Opt. Soc. Am. A 12, 2358-2366 (1995).

[9] P. Artal, and R. Navarro, "Monochromatic modulation transfer function of the human eye for different pupil diameters: an analytical expression," J. Opt. Soc. Am. A 11, 246-249 (1994).

[10] J. A. Martínez-Roda, M. Vilaseca, J. C. Ondategui, A. Giner, F. J. Burgos, G. Cardona, and J. Pujol, "Optical quality and intraocular scattering in a healthy young population," Clin. Exp. Optom. 94, 223229 (2011).

[11] P. Artal, A. Benito, G. M. Pérez, E. Alcón , A. De Casas, J. Pujol, and J. M. Marín, "An objective scatter index based on double-pass retinal images of a point source to classify cataracts," PLOS One 6, e16823 (2011). 